\title{
Pseudoaneurisma de la arteria uterina post cesárea.
}

\section{Pseudoaneurysm of the uterine artery after}

\section{cesarean.}

\begin{abstract}
RESUMEN
El pseudoaneurisma de la arteria uterina es poco habitual pero una grave complicación de la cesárea, puede manifestarse con hemorragia de importancia clínica durante el postoperatorio tardío. La ecografía doppler fue un procedimiento importante para su sospecha diagnóstica del pseudoaneurisma la misma que puede tratarse por medio de la embolización arteriográfica de la arteria uterina. Comunicamos el caso de una paciente con 17 días de hemorragia post cesárea sometida a legrado uterino y 21 días después del legrado se le realiza histerectomía con el diagnóstico de pseudoaneurisma de arteria uterina.
\end{abstract}

Palabras clave: Falso aneurisma, arteria uterina, hemorragia uterina, ultrasonografía doppler. Perú (Fuente: DeCS BIREME).

\section{ABSTRACT}

The pseudoaneurysm of the uterine artery is uncommon but a serious complication of caesarean section may manifest with hemorrhage clinical importance during the late postoperative period. Doppler ultrasound was an important procedure for its diagnostic suspicion of the pseudoaneurysm, which can be treated by arteriographic embolization of the uterine artery. We report the case of a patient with 17 days of post-cesarean haemorrhage submitted to uterine curettage and 21 days after curettage a hysterectomy was performed with the diagnosis of pseudoaneurysm of the uterine artery.

Keywords: Aneurysm false, uterine artery, uterine hemorrhage, Ultrasonography doppler. Peru (Source: MeSH NLM)

\footnotetext{
Correspondencia:

Ybaseta-Soto George Gino Dirección: Urb. Villa del Médico B-7, Ica, Perú. Número de celular: 999613922 Correo Electrónico: georgeginoybaseta@outlook.com

Contribuciones de autoría:

YSG, PFG, PSE, NRP participaron en la recolección de datos, diseño, búsqueda de bibliografía, interpretación, redacción y aprobación de la versión final.

Como citar:

George Ybaseta-Soto, Gustavo Alonso Palacios-Flores, Estefany Pachas-Serpa, Pierina Claudia Napa Ruiz. Caso clínico de un
} Pseudoaneurisma de la arteria uterina post cesárea. Rev méd panacea. 2018;7(1):34-37 


\section{INTRODUCCIÓN}

El pseudoaneurisma de la arteria uterina es una rara pero calificada como una grave complicación después de la cesárea y puede manifestarse como hemorragia importancia clínica durante el postoperatorio.

El pseudoaneurisma es poco frecuente y se asocia a complicaciones de cirugías, parto vaginal, neoplasias, traumatismos e infecciones. El pseudoaneurisma de arteria uterina es una de las causas de hemorragia en el puerperio poco conocida, está asociada a procedimientos ginecoobstétricos que ocasionan lesión del vaso uterino y que causan hemorragias intermitentes graves y suelen ser tardías respecto al acto quirúrgico (1).

Este problema que ocurre en la arteria no está muy documentada, se desarrolla cuando la arteria uterina es lacerada o lesionada ocurre extravasación de sangre y migra a través de los tejidos, estableciendo finalmente una conexión con la cavidad uterina, causando una hemorragia retardada (2).

Ante hemorragia persistente posterior a la cesárea la ecografía doppler puede dar información de la sospecha de pesudoaneurisma, la angiografía pélvica permite el diagnóstico correcto y la terapéutica adecuada en el mismo procedimiento, mediante una técnica de bajo riesgo, con conservación de la fertilidad (3), alta tasa de efectividad en el control de la hemorragia puerperal y prevención de la morbilidad y mortalidad materna asociadas con las clásicas cirugías mayores (1).

El objetivo de esta comunicación es presentar un caso no habitual que al menos no se encuentran reportes publicados de pseudoaneurisma de la arteria uterina en nuestra Región Ica, Perú.

\section{PRESENTACIÓN DEL CASO}

Paciente de 40 años de edad, multipara, cesáreada por sufrimiento fetal agudo, acude a los 17 días de la intervención quirúrgica a una clínica de su localidad por presentar hemorragia genital excesiva, donde se le realizó legrado evacuador por tener imagen ecográfica de cavidad uterina de contenido heterogéneo. Fue dada de alta 24 horas después con escaso sangrado.

21 días después de realizarse el legrado uterino es traída al servicio de emergencias del Hospital de su localidad por presentar hemorragia genital con expulsión de coágulos, signos de shock hipovolémico, dolor abdominal en hipogastrio tipo cólico de moderada intensidad.

\section{- Al ingreso:}

Funciones vitales:

\section{P.A: $70 / 40 \mathrm{mmhg}$} pulso: $114 x^{\prime}$

FR:22 $x^{\prime}$

Afebril
Datos de laboratorio: Anemia grave $(\mathrm{Hb} 7.8 \mathrm{~g} / \mathrm{dL}$, hematocrito $24 \%$ ) con pruebas de coagulación ( Tiempo de coagulación 7'20", Tiempo de sangría 2'30"); leucocitos:15 800; plaquetas: 410000; urea:16; creatinina: 0.8; glucosa: $177 \mathrm{mg} / \mathrm{dl}$ ).

Se procede a la administración 2 paquetes globulares $(O$ $\mathrm{Rh}+$ ), cristaloides, coloides y oxitocina más masaje uterino. Una vez controlado el cuadro se procede a su hospitalización.

\section{- Se realiza ecografía al primer día de hospitalización de servicio de Ginecología}

Útero: Retroversión de eco estructura homogéneo, bordes regulares que miden $85 \mathrm{~mm}$ de longitud por $48 \mathrm{~mm}$ anteroposterior. Cavidad endometrial de $1.2 \mathrm{~cm}$. Al nivel del tercio inferior de útero se observa una imagen redondeada isoecogénico anecoicos de $9 \mathrm{~mm}$. Anexos: características normales Figura 1 y 2.

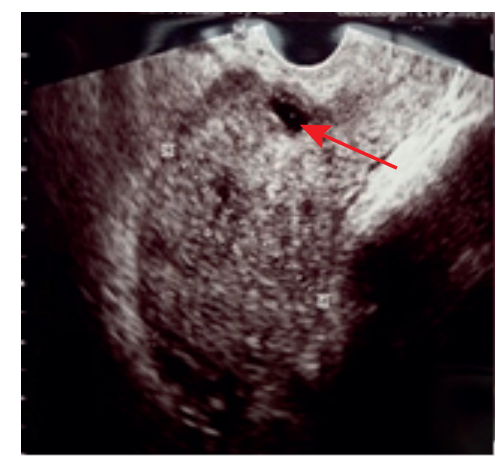

Figura 1. Psuedoaneurisma

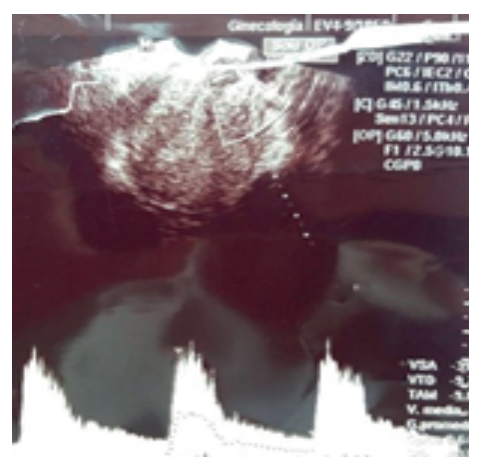

Figura 2. Doppler pseudoaneurisma

\section{- Tomografía Axial Computarizada de abdomen inferior sin contraste al segundo día de hospitalización:}

Útero: incrementado de tamaño central parénquima heterogéneo, anexos sin alteraciones, no evidencia de lesiones solidas ni quísticas en la fosa pélvica. Vasos iliacos muestran adecuada calibre, no se evidencian adenomegalias.

No se aprecia lesiones transmurales en el tracto genito urinario, no lesiones quísticas, ni masas en cavidad pélvica, ausencia de líquido libre en cavidad peritoneal. 
Vejiga: distendida de paredes delgadas, no se evidencia engrosamiento patológico focal ni difuso de sus paredes, ni alteración de grasa perivesical Figura 3.

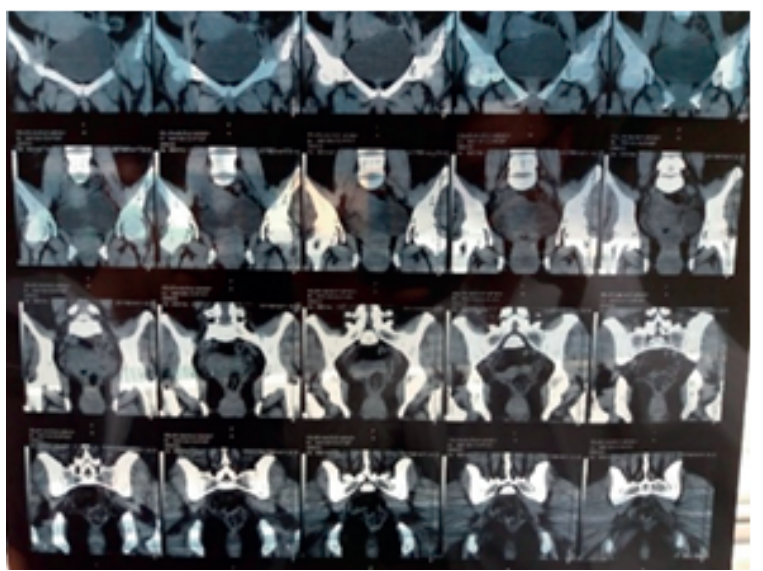

Figura 3. TAC útero aumentado de tamaño

\section{- Nuevamente se repite el examen ecografía doppler en el servicio de Radiología al 4to día de hospitalización:}

Vejiga: con poca distención, paredes delgadas contenido anecogénico. Útero: en anteversoflexión, de dimensiones $108 \times 41 \times 59 \mathrm{~mm}$. Parénquima homogéneo a nivel de pared anterior en herida operatoria se aprecia imagen anecogénico de $13 \times 10 \mathrm{mmm}$ de diámetro que al doppler presenta el signo de yin-yang, en relación a un pseudoaneurisma. Endometrio: ecogénico laminar $7 \mathrm{~mm}$. Anexos: Ovarios: de morfología conservada. Fondo de saco: No líquido libre Figura 4.

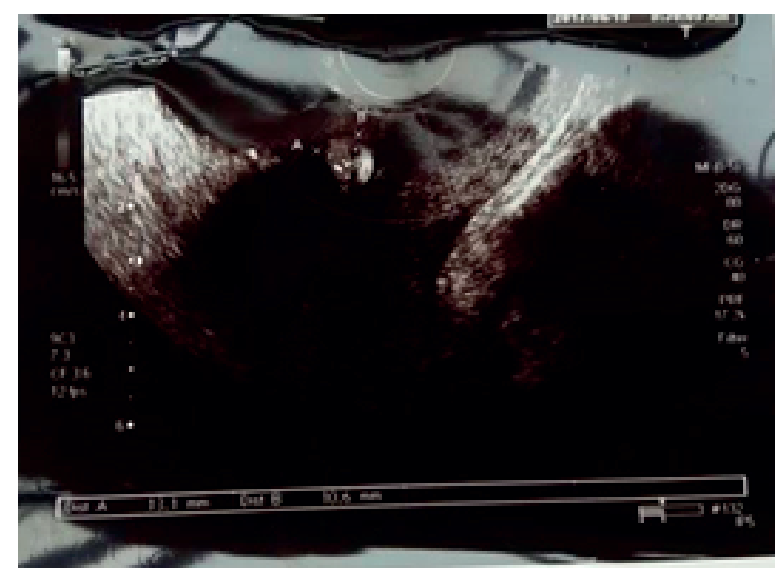

Figura 4. Signo de yin yang en el interior del saco de pseudoaneurisma

\section{DISCUSIÓN}

La hemorragia posparto constituye una importante causa de morbilidad y mortalidad materna en el mundo siendo las principales causas la atonía uterina, retención de restos placentarios y entre los menos frecuentes esta lo que reportamos (1).

La historia natural del pseudoaneurisma no está debidamente documentada los autores coinciden que se produce cuando la arteria uterina está lacerada o lesionada. Mientras se mantiene el contacto con el vaso progenitor, la sangre extravasante disecciona a través de los tejidos, estableciendo finalmente una conexión con la cavidad uterina, lo que provoca una hemorragia retrasada $(1,3)$.

El caso que presentamos fue una hemorragia intermitente que persistió varias semanas que puso en riesgo la vida de la madre, casos parecidos han sido reportados en donde la sospecha diagnóstica es primero en las causas más frecuentes de hemorragia puerperal tardía $(1,2)$.

La ecografía para nosotros ha sido el procedimiento de ayuda diagnóstica más importante sin embargo para otros centros de alta especialización es la arteriografía como indican diversos autores que se refieren al tratamiento del pseudoaneurisma relacionada a la arteria uterina $(3,5$,$) .$

La ecografía doppler es un procedimiento que en estos tiempos es accesible y no provoca daño a la paciente, el resultado de la ecografía fue informado por los médicos del servicio de ginecología y radiología del Hospital como una imagen característica de un pseudoaneurisma, describiéndola como una imagen anecoica, redondeada, bien delimitada como informan otros autores y al doppler según el informe el signo del yin-yang que orientó al diagnóstico $(1,2)$.

La ultrasonografía con Doppler puede establecer el diagnóstico en forma rápida del pseudoaneurisma de la arteria uterina mientras que la Tomografía Axial Computarizada siendo una tecnología con que se espera tener mejor resultado no ayudó al diagnóstico.

La arteriografía es el método de elección para el diagnóstico porque permite el tratamiento concomitante mediante la embolización arterial selectiva el inconveniente es que esta técnica no está disponible en todos los Hospitales $(4,5)$, en nuestro caso se le realizó el tratamiento de Histerectomía Abdominal debido al estado hemodinámico de la paciente, y a los deseos genésicos cumplidos.

\section{CONCLUSIÓN}

Ante una paciente post-cesárea que presenta sangrado uterino de consideración clínica importante y la ecografía sea compatible con pseudoaneurisma debe considerarse la arteriografía más embolización o el procedimiento conveniente que evite la hemorragia.

- Consentimiento del paciente: La paciente autoriza plenamente la publicación de su caso.

- Agradecimientos: Damos las gracias a los Ginecólogos del Hospital Santa María del Socorro de Ica, Perú, por apoyar al desarrollo de este reporte de caso. 


\section{REFERENCIAS BIBLIOGRÁFICAS}

1. Pérez B, Carazo B, Arribas-T, Guardia-Dodorico. Pseudoaneurisma de arteria uterina después de una cesárea. Ginecol Obstet Mex 2013;81:166-170

2. Brian Cooper, Hocking-Brown, Joel I Sorosky and Wendy $F$ Hansen. Pseudoaneurysm of the Uterine Artery Requiring Bilateral Uterine Artery Embolization. Journal of Perinatology (2004) 24, 560-562.

https://www.nature.com/articles/7211119

3. López V Nuria, Vegas G Gabriel, Arrieta B Sara, Iglesias S Carlos, González G Antonio. Reembolización de arterias uterinas por hemorragia posparto en una segunda gestación. Rev. chil. obstet. ginecol. [Internet]. 2012 [citado 2018 Abr 30] ; 77(1): 40-43. Disponible en:

https://scielo.conicyt.cl/scielo.php?script=sci_arttext $\&$ pid=S0717-75262012000100008\&lng =es.

http://dx.doi.org/10.4067/S0717-7526201200010000 8.
4. Descargues G, Douvrin F, Gravier A, Lemoine JP, Marpeau L, Clavier E. False aneurysm of the uterine pedicle: an uncommon cause of post-partum haemorrhage after caesarean section treated with selective arterial embolization. Eur J Obstet Gynecol Reprod Biol. 2001;97(1):26-9 https://www.ncbi.nlm.nih.gov/pubmed/11435004

5. Kim JW, et al. Uterine artery pseudoaneurysm manifesting as delayed postpartum hemorrhage after precipitous delivery: three case reports. Gynecol Obstet Invest 2014; 78:136-140 https://www.ncbi.nlm.nih.gov/m/pubmed/25012906 /

\section{Las ediciones anteriores de revista médica PANACEA están disponibles en:}

\section{www.revpanacea.unica.edu.pe}

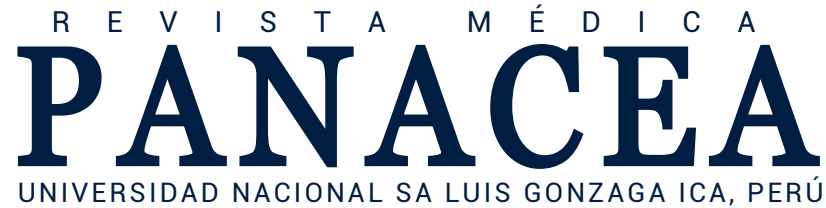

